\title{
Rank 2 Ulrich bundles on general double plane covers
}

Ronnie Sebastian $^{\mathrm{a}, *}$, Amit Tripathi ${ }^{\mathrm{b}}$

a Department of Mathematics, Indian Institute of Technology Bombay, Powai, Mumbai 400076,

Maharashtra, India

b Department of Mathematics, Indian Institute of Technology Hyderabad, Kandi, Sangareddy, 502285,

Telangana, India

\section{A R T I C L E I N F O}

\section{Article history:}

Received 4 December 2020

Received in revised form 2 May 2021

Available online 19 June 2021

Communicated by V. Suresh

\section{$M S C$ :}

$14 \mathrm{E} 20 ; 14 \mathrm{~J} 60 ; 14 \mathrm{H} 50$

Keywords:

Ulrich bundles

Double planes

Cayley-Bacharach

\section{A B S T R A C T}

We prove that a double cover of $\mathbb{P}^{2}$ ramified along a general smooth curve $B$ of degree $2 s$, for $s \geq 3$, supports a rank 2 special Ulrich bundle.

() 2021 Elsevier B.V. All rights reserved.

\section{Introduction}

Throughout this paper we work over the field of complex numbers $\mathbb{C}$. Let $X$ be a $d$-dimensional smooth projective variety. Unless mentioned otherwise, $\mathcal{O}_{X}(1)$ will always denote an ample and globally generated line bundle on $X$.

Definition 1.1. A locally free sheaf (vector bundle) $E$ on $X$ is said to be Ulrich with respect to $\mathcal{O}_{X}(1)$ (or simply Ulrich when the bundle $\mathcal{O}_{X}(1)$ is understood) if the following two conditions are satisfied

(1) $H^{i}(X, E(-i))=0$ for all $i>0$,

(2) $H^{j}(X, E(-j-1))=0$ for all $j<n$.

We refer the reader to [3, §2] for basic definitions. In the literature authors usually define Ulrich with respect to a very ample line bundle, kindly see the next section for some remarks related to this. A conjecture

\footnotetext{
* Corresponding author.

E-mail addresses: ronnie@math.iitb.ac.in (R. Sebastian), amittr@gmail.com (A. Tripathi).
} 
of Eisenbud and Schreyer [11] states that every smooth projective variety supports an Ulrich bundle. Several people have constructed Ulrich bundles on particular varieties and we list a few. They have been shown to exist on complete intersections by [14], on curves and del Pezzo surfaces by [11], on $K 3$ surfaces by [2] and [12], on abelian surfaces by [4], on ruled surfaces by [1], on regular surface by [6], [8], on surfaces with $p_{g}=0$ and $q=1$ by [7], on surfaces of maximal Albanese dimension and some irregular surfaces by [15], [16]. The above list is far from being complete and we refer the reader to the above papers, for example [7], and the references therein for more results. In [10, Theorem 4.3] the authors show, over the field of complex numbers, the existence of Ulrich bundles of rank two in a sufficiently ample embedding.

Recently Narayanan and Parameswaran [17] studied the existence of Ulrich line bundles on a double plane $\pi: X \rightarrow \mathbb{P}^{2}$ branched along a smooth curve $B \subset \mathbb{P}^{2}$ of degree $2 s$. In [17, Theorem 1.5], they prove that for each $s \geq 3$, there are special classes of double planes which admit Ulrich line bundles. In [17, Theorem 1.4] they show that a double plane branched along a generic smooth curve of degree $2 s$, where $s \geq 3$, does not support an Ulrich line bundle.

Let $X$ be a surface and let $K_{X}$ be the canonical line bundle. An Ulrich bundle $E$ of rank 2, with respect to $\mathcal{O}_{X}(1)$, is called special Ulrich if it also satisfies $\operatorname{det}(E) \cong K_{X} \otimes \mathcal{O}_{X}(3)$. Let $\pi: X \rightarrow \mathbb{P}^{2}$ be a degree 2 cover which is branched along a smooth curve $B \subset \mathbb{P}^{2}$ of degree $2 s$. For such a map denote $\mathcal{O}_{X}(1):=\pi^{*} \mathcal{O}_{\mathbb{P}^{2}}(1)$ and by an Ulrich (respectively, special Ulrich) bundle on $X$ we will always mean Ulrich (respectively, special Ulrich) with respect to $\mathcal{O}_{X}(1)$. In this note we show the following.

Theorem 1.2. Let $\pi: X \rightarrow \mathbb{P}^{2}$ be a double cover branched along a generic smooth curve $B \subset \mathbb{P}^{2}$ of degree $2 s$, where $s \geq 3$. Then $X$ admits a special rank 2 Ulrich bundle.

To prove the above result we use two inputs. The first is the well known correspondence between zero dimensional subschemes satisfying the Cayley-Bacharach property and global sections of a rank 2 vector bundle, see $[19, \S 5]$. Let $F$ be the degree $2 s$ homogeneous polynomial which defines $B$. Using [19] we first prove

Theorem 1.3 (Theorem 2.7). Let $\pi: X \rightarrow \mathbb{P}^{2}$ be a degree 2 cover which is branched along a smooth curve $B \subset \mathbb{P}^{2}$ of degree $2 s$, where $s \geq 3$. Let $F$ denote the polynomial of degree $2 s$ which defines $B$. Assume that there are two polynomials $F_{1}$ and $F_{2}$ of degree $s$ such that $F \in\left(F_{1}, F_{2}\right)$. Then $X$ supports a special Ulrich bundle of rank 2.

The second input is the first point in [9, Theorem 5.1] which enables us to conclude that for the general degree $2 s$ hypersurface $F$ we can find degree $s$ hypersurfaces $F_{1}$ and $F_{2}$ such that $F \in\left(F_{1}, F_{2}\right)$. We do not know if this holds for all smooth degree $2 s$ hypersurfaces.

It has been brought to our attention that Mohan Kumar, P. Narayanan and A.J. Parameswaran have proved, using a different method, that every double plane cover supports a rank 2 Ulrich bundle.

Acknowledgements. We thank Enrico Carlini and Luca Chiantini for several helpful discussions related to their article [9]. We thank Gianfranco Casnati for several useful comments. We thank the referee for an extremely careful reading of this article and for numerous useful suggestions which have improved the exposition.

\section{Existence of Ulrich bundles}

To show that a bundle $E$ on $X$ is Ulrich we will use the following criterion.

Lemma 2.1. Let $X$ be a d-dimensional smooth projective variety and let $\pi: X \rightarrow \mathbb{P}^{d}$ be a surjective and finite map of degree e. A bundle $E$ on $X$ is Ulrich with respect to $\pi^{*} \mathcal{O}_{\mathbb{P}^{d}}(1)$ if and only if $\pi_{*} E \cong \mathcal{O}_{\mathbb{P}^{d}}^{e \cdot \operatorname{rank}(E)}$. 
Proof. Let us first assume that $\pi_{*} E \cong \mathcal{O}_{\mathbb{P}^{d}}^{e \operatorname{rank}(E)}$. Since the map is finite, and using projection formula, we have $H^{i}(X, E(k))=H^{i}\left(\mathbb{P}^{d}, \pi_{*} E(k)\right)$ for all $i, k \in \mathbb{Z}$. Since $\pi_{*} E \cong \mathcal{O}_{\mathbb{P} d}^{e \operatorname{rank}(E)}$ it is clear that the conditions in Definition 1.1 are satisfied.

Conversely, assume that $E$ satisfies the conditions in Definition 1.1. Then it follows that $\pi_{*} E$ and $\left(\pi_{*} E\right)^{\vee}$ are 0-regular of rank $e \cdot \operatorname{rank}(E)$. Thus, both of them are $m$-regular for all $m \geq 0$. From this it easily follows that $H^{i}\left(\mathbb{P}^{d}, \pi_{*} E(k)\right)=0$ for all $k \in \mathbb{Z}$ and for all $1 \leq i \leq d-1$, that is, $\pi_{*} E$ is an ACM bundle. Now applying Horrocks criterion, see [13] or [18, Theorem 2.3.1] where it is stated more precisely, we get that $\pi_{*} E$ is a direct sum of line bundles. If $\mathcal{O}_{\mathbb{P}^{d}}(a)$ is a summand of $\pi_{*} E$ then we get that $H^{i}\left(\mathbb{P}^{d}, \mathcal{O}_{\mathbb{P}^{d}}(a-i)\right)=0$ for all $i>0$ and $H^{j}\left(\mathbb{P}^{d}, \mathcal{O}_{\mathbb{P}^{d}}(a-j-1)\right)=0$ for all $j<d$. In particular, by taking $i=d$ and $j=0$ it follows that $a=0$. This shows that $\pi_{*} E \cong \mathcal{O}_{\mathbb{P}^{d}}^{e \cdot \operatorname{rank}(E)}$.

As mentioned before, authors usually define Ulrich bundles with respect to very ample line bundles. However, the existence of an Ulrich bundle with respect to an ample and globally generated line bundle $L$ ensures that there are Ulrich bundles with respect to $L^{\otimes n}$ for all $n>0$, see [3, Proposition 3] and the remarks following it.

2.2. Double covers of $\mathbb{P}^{2}$. We briefly recall the main properties of double covers which we will use. A general reference for this is $[5, \S 17]$. Let $B \subset \mathbb{P}^{2}$ be a smooth curve of degree $2 s$ defined by a homogeneous polynomial $F$. Let $\pi: X \rightarrow \mathbb{P}^{2}$ be the double cover of $\mathbb{P}^{2}$ branched along $B$, the construction of which we briefly explain for the benefit of the reader. Let $\mathbb{A}$ denote the total space of the line bundle $A=\mathcal{O}_{\mathbb{P}^{2}}(s)$, $\pi: \mathbb{A} \rightarrow \mathbb{P}^{2}$ the projection and

$$
T \in H^{0}\left(\mathbb{A}, \pi^{*} A\right)
$$

be the tautological section. Define $X$ to be the subvariety of $\mathbb{A}$ defined by the section $T^{2}-\pi^{*} F \in$ $H^{0}\left(\mathbb{A}, \pi^{*} A^{\otimes 2}\right)=H^{0}\left(\mathbb{A}, \pi^{*} \mathcal{O}_{\mathbb{P}^{2}}(2 s)\right)$. We will abuse notation and denote the composite $X \subset \mathbb{A} \rightarrow \mathbb{P}^{2}$ also by $\pi$. Then $\pi$ is a finite map of degree 2 between projective varieties. We list the important properties of double covers that we will use, see [5, Lemma 17.1, Lemma 17.2].

(1) If $B$ is smooth then $X$ is smooth, this is explained in the sentence preceding [5, Lemma 17.1].

(2) Let $R \subset X$ denote the reduced divisor $\pi^{-1}(B)$. Then $\pi^{*} \mathcal{O}_{\mathbb{P}^{2}}(s) \cong \mathcal{O}_{X}(R)$.

(3) The canonical bundle $K_{X} \cong \mathcal{O}_{X}(s-3)$.

(4) $\pi_{*} \mathcal{O}_{X}=\mathcal{O}_{\mathbb{P}^{2}} \oplus \mathcal{O}_{\mathbb{P}^{2}}(-s)$.

2.4. Existence of Ulrich bundles. In this section we shall prove that a general double plane cover supports a special Ulrich bundle of rank 2. We will use the result in [19, Theorem 10] to construct a rank 2 bundle on $X$. For the benefit of the reader we state the main result from [19] that we need, but first we recall some definitions from [19, page 2]. Given a subscheme $Z_{2} \subset Z_{1}$, the "complement" $Z$ of $Z_{2}$ in $Z_{1}$ is the canonical closed subscheme $Z \subset Z_{1}$ with sheaf of ideals $I_{Z}=\left[I_{Z_{1}}: I_{Z_{2}}\right]$, that is, for any open set $U \subset X$, we define

$$
I_{Z}(U):=\left\{g \in \mathcal{O}_{X}(U) \mid g I_{Z_{2}}(U) \subset I_{Z_{1}}(U)\right\}
$$

We call $Z$ the residual subscheme of $Z_{2}$ in $Z_{1}$ and denote it by $Z=Z_{1}-Z_{2}$ in the statement of the next theorem, which is [19, Theorem 10]. There are three equivalences, but we state only two of these.

Theorem 2.5 (Theorem 10, [19]). Let $X$ be a complex smooth projective variety of dimension $n \geq 2$. Let $Z \subset X$ be a subscheme of pure codimension 2. Then the following are equivalent:

(1) $Z$ is the zero subscheme of a section of a rank 2 vector bundle $\mathcal{E}$. 
(2) There are hypersurfaces $D_{1}, D_{2}, D_{3}$ such that $D_{1}$ and $D_{2}$ have no common components, $Z=D_{1} \cap D_{2}-$ $D_{1} \cap D_{2} \cap D_{3}$ and such that $D_{1} \cap D_{2} \cap D_{3}$ is of pure codimension 2 and is Cohen-Macaulay.

Further, if (1) and (2) hold then $\operatorname{det}(\mathcal{E}) \equiv \mathcal{O}_{X}\left(D_{1}+D_{2}-D_{3}\right)$.

With notation as above let $\mathcal{E}$ be the bundle which sits in the following short exact syzygy sequence, this is explained just before [19, Theorem 10].

$$
0 \rightarrow \mathcal{E}\left(-D_{1}-D_{2}\right) \rightarrow \bigoplus_{i} \mathcal{O}_{X}\left(-D_{i}\right) \rightarrow I_{D_{1} \cap D_{2} \cap D_{3}} \rightarrow 0
$$

Theorem 2.7. Let $\pi: X \rightarrow \mathbb{P}^{2}$ be a degree 2 cover which is branched along a smooth curve $B \subset \mathbb{P}^{2}$ of degree $2 s$, where $s \geq 3$. Let $F$ denote the polynomial of degree $2 s$ which defines $B$. Assume that there are two polynomials $F_{1}$ and $F_{2}$ of degree s such that $F \in\left(F_{1}, F_{2}\right)$. Then $X$ supports a special Ulrich bundle of rank 2.

Proof. First let us note that there is no non-constant polynomial $H$ which divides both $F_{1}$ and $F_{2}$, or else $H$ will divide $F$, contradicting the smoothness of $B$. Thus, the subscheme of $\mathbb{P}^{2}$ defined by the ideal $\left(F_{1}, F_{2}\right)$ is 0 -dimensional and is contained in $B$. We denote this by $Z^{\prime}$ the subscheme defined by the ideal $\left(F_{1}, F_{2}\right)$. Consider the scheme theoretic inverse image $Z_{1}:=\pi^{-1}\left(Z^{\prime}\right)$.

For $i=1,2$ take $H_{i}=\pi^{*} F_{i} \in H^{0}\left(X, \mathcal{O}_{X}(s)\right)$. If $D_{i}$ denotes the divisor defined by $H_{i}$ then $Z_{1}=D_{1} \cap D_{2}$ in the notation of Theorem 2.5. Take $H_{3}=T \in H^{0}\left(X, \mathcal{O}_{X}(s)\right)$ (see equation (2.3), by abuse of notation we denote the restriction of $T$ to $X$ also by $T$ ) and $Z_{2}$ to be the subscheme of $Z_{1}$ defined by $H_{3}$, thus, $Z_{2}=D_{1} \cap D_{2} \cap D_{3}$, where $D_{3}$ is the divisor defined by $H_{3}$. Let us compute the ideal $I_{Z}:=\left[I_{Z_{1}}: I_{Z_{2}}\right]$.

If $x, y, z$ are homogeneous coordinates on $\mathbb{P}^{2}$ then let $\mathbb{C}[x, y]$ (we abuse notation here and denote the affine coordinates also by $x$ and $y$ ) denote the coordinate ring of the open set $\{z \neq 0\}$. Denote by $f$ the equation $F$ in $\mathbb{C}[x, y]$, similarly, for the other polynomials. The inverse image of this open set in $X$ has coordinate ring

$$
\mathbb{C}[x, y, t] /\left(t^{2}-f\right) \text {. }
$$

The ideal $I_{Z_{1}}=\left(f_{1}, f_{2}\right)$ and the ideal $I_{Z_{2}}=\left(f_{1}, f_{2}, t\right)$. If $I \subset J$ are two ideals in a ring then it is clear that $I \subset[I: J]$. Thus, $I_{Z_{1}} \subset I_{Z}$. We claim that $t \in\left[I_{Z_{1}}: I_{Z_{2}}\right]$. An element of $I_{Z_{2}}$ looks like $\lambda f_{1}+\mu f_{2}+\theta t$. Since $t^{2}=f \in\left(f_{1}, f_{2}\right)$ it follows that $t\left(\lambda f_{1}+\mu f_{2}+\theta t\right) \in I_{Z_{1}}$ that is, $t \in\left[I_{Z_{1}}: I_{Z_{2}}\right]$. Thus, $I_{Z}=\left(f_{1}, f_{2}, t\right)$. In particular, $Z=Z_{2}=D_{1} \cap D_{2} \cap D_{3}$. Thus, in the notation from [19, §1, page 2], we may write

$$
Z=D_{1} \cap D_{2}-D_{1} \cap D_{2} \cap D_{3}
$$

Moreover, $D_{1} \cap D_{2} \cap D_{3}$ is of pure codimension 2 and is Cohen-Macaulay (since both depth and dimension are 0 ). Thus, there is a bundle $\mathcal{E}$ which sits in the short exact sequence (2.6) and has a global section

$$
\mathcal{O}_{X} \rightarrow \mathcal{E}
$$

whose vanishing gives $Z$. The line bundles $\mathcal{O}_{X}\left(D_{i}\right)$ are all isomorphic to $\mathcal{O}_{X}(s)$. Thus, $\operatorname{det}(\mathcal{E})=\mathcal{O}_{X}(s)$. Twisting the short exact sequence $(2.6)$ by $\mathcal{O}_{X}(2 s)$ we get a short exact sequence

$$
0 \rightarrow \mathcal{E} \rightarrow \mathcal{O}_{X}(s)^{\oplus 3} \rightarrow I_{Z}(2 s) \rightarrow 0
$$

Consider the commutative diagram 


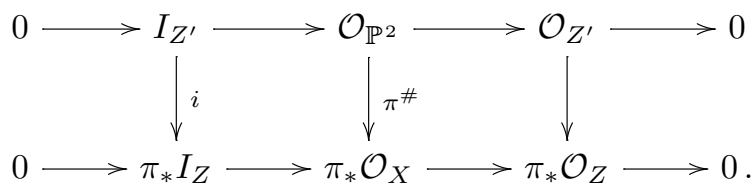

In the notation we used above, the coordinate ring of $Z^{\prime}$ is $\mathbb{C}[x, y] /\left(f_{1}, f_{2}\right)$ and the coordinate ring of $Z$ is $\mathbb{C}[x, y, t] /\left(f_{1}, f_{2}, t\right) \cong \mathbb{C}[x, y] /\left(f_{1}, f_{2}\right)$. Thus, the right vertical arrow is an isomorphism, which proves that the cokernel of $\pi^{\#}$ and $i$ are isomorphic.

Let $\sigma$ denote the involution of $X$ interchanging the two sheets of the double cover. One has the trace map $\operatorname{Tr}: \pi_{*} \mathcal{O}_{X} \rightarrow \mathcal{O}_{\mathbb{P}^{2}}$ defined as follows. Let $U \subset \mathbb{P}^{2}$ be open and let $f \in \mathcal{O}_{X}\left(\pi^{-1}(U)\right)$. Define $\operatorname{Tr}(f):=$ $f+f \circ \sigma \in \mathcal{O}_{\mathbb{P}^{2}}(U)$. Now if $f \in \mathcal{O}_{X}\left(\pi^{-1}(U)\right)$ and $f$ vanishes at a point $p \in Z$, then since $Z \subset B$ and $\sigma$ is the identity on $B$, it follows that $f \circ \sigma$ also vanishes at $p$. From this it is clear that Tr maps $\pi_{*} I_{Z}$ to $I_{Z^{\prime}}$.

The map Tr gives a splitting of the map $\pi^{\#}$. Since Tr maps $\pi_{*} I_{Z}$ to $I_{Z^{\prime}}$ it follows that $\operatorname{Tr}$ gives a splitting of the map $i$. Thus, $\pi_{*} I_{Z}$ is isomorphic to the direct sum of $I_{Z^{\prime}}$ and the cokernel of $i$. We saw above that the cokernel of $\pi^{\#}$ and the cokernel of $i$ are isomorphic. Since $\pi_{*} \mathcal{O}_{X}=\mathcal{O}_{\mathbb{P}^{2}} \oplus \mathcal{O}_{\mathbb{P}^{2}}(-s)$, see property (4) in subsection 2.2, it follows that the cokernel of $\pi^{\#}$ is isomorphic to $\mathcal{O}_{\mathbb{P}^{2}}(-s)$. Thus, it follows that

$$
\pi_{*} I_{Z} \cong I_{Z^{\prime}} \oplus \mathcal{O}_{\mathbb{P}^{2}}(-s)
$$

Thus, we have

$$
h^{0}\left(X, I_{Z}(2 s)\right)=h^{0}\left(\mathbb{P}^{2}, I_{Z^{\prime}}(2 s)\right)+h^{0}\left(\mathbb{P}^{2}, \mathcal{O}_{\mathbb{P}^{2}}(s)\right) .
$$

Using the short exact sequence

$$
0 \rightarrow \mathcal{O}_{\mathbb{P}^{2}}(-2 s) \rightarrow \mathcal{O}_{\mathbb{P}^{2}}(-s)^{\oplus 2} \rightarrow I_{Z^{\prime}} \rightarrow 0,
$$

we get that $h^{0}\left(\mathbb{P}^{2}, I_{Z^{\prime}}(2 s)\right)=2 h^{0}\left(\mathbb{P}^{2}, \mathcal{O}_{\mathbb{P}^{2}}(s)\right)-1$. Using equation $(2.11)$ we get

$$
h^{0}\left(X, I_{Z}(2 s)\right)=3 h^{0}\left(\mathbb{P}^{2}, \mathcal{O}_{\mathbb{P}^{2}}(s)\right)-1 .
$$

Next we will compute $H^{0}(X, \mathcal{E})$. Taking dual of $(2.8)$ we get an exact sequence

$$
0 \rightarrow \operatorname{det}(\mathcal{E})^{\vee} \rightarrow \mathcal{E}^{\vee} \rightarrow I_{Z} \rightarrow 0
$$

Since $\operatorname{det}(\mathcal{E})=\mathcal{O}_{X}(s)$ and $\mathcal{E}$ is of rank 2 , we get $\mathcal{E}^{\vee}=\mathcal{E} \otimes \operatorname{det}(\mathcal{E})^{\vee}$, which gives

$$
0 \rightarrow \mathcal{O}_{X} \rightarrow \mathcal{E} \rightarrow I_{Z}(s) \rightarrow 0
$$

Applying $\pi_{*}$ to this we get

$$
0 \rightarrow \pi_{*} \mathcal{O}_{X} \rightarrow \pi_{*} \mathcal{E} \rightarrow \pi_{*} I_{Z}(s) \rightarrow 0
$$

Using (2.12) we get $h^{0}\left(\mathbb{P}^{2}, I_{Z^{\prime}}(s)\right)=2$ and using (2.10) we get that

$$
h^{0}\left(\mathbb{P}^{2}, \pi_{*} I_{Z}(s)\right)=3
$$

From this it follows that $h^{0}\left(\mathbb{P}^{2}, \pi_{*} \mathcal{E}\right)=4$. Applying $\pi_{*}$ to (2.9) and taking cohomology we get 


$$
\begin{aligned}
h^{1}\left(\mathbb{P}^{2}, \pi_{*} \mathcal{E}\right) & =h^{0}\left(\mathbb{P}^{2}, \pi_{*} I_{Z}(2 s)\right)+h^{0}\left(\mathbb{P}^{2}, \pi_{*} \mathcal{E}\right)-3-3 h^{0}\left(\mathbb{P}^{2}, \mathcal{O}_{\mathbb{P}^{2}}(s)\right) \\
& =3 h^{0}\left(\mathbb{P}^{2}, \mathcal{O}_{\mathbb{P}^{2}}(s)\right)-1+4-3-3 h^{0}\left(\mathbb{P}^{2}, \mathcal{O}_{\mathbb{P}^{2}}(s)\right) \\
& =0 .
\end{aligned}
$$

Consider the commutative diagram

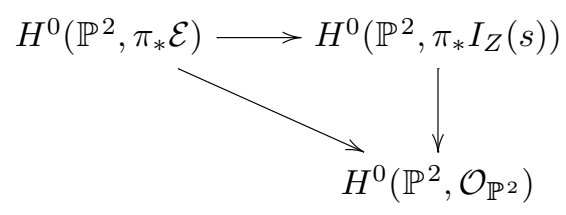

The vertical arrow is the projection in equation (2.10) and is surjective. The horizontal arrow is surjective as is easily seen by taking cohomology of the sequence (2.13). Thus, we have a map $\pi_{*} \mathcal{E} \rightarrow \mathcal{O}_{\mathbb{P}^{2}}$ which induces a surjection on global sections. This shows that this map is split. Thus,

$$
\pi_{*} \mathcal{E}=\mathcal{G} \oplus \mathcal{O}_{\mathbb{P}^{2}}
$$

where $\mathcal{G}$ is a locally free sheaf and sits in a short exact sequence

$$
0 \rightarrow \pi_{*} \mathcal{O}_{X} \rightarrow \mathcal{G} \rightarrow I_{Z^{\prime}}(s) \rightarrow 0
$$

We will now show that $\mathcal{G}$ is trivial. Consider the following pullback diagram.

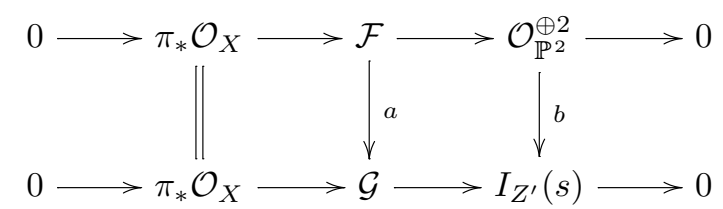

From this it follows that $\mathcal{F}=\mathcal{O}_{\mathbb{P}^{2}}^{\oplus 3} \oplus \mathcal{O}_{\mathbb{P}^{2}}(-s)$ since $\operatorname{Ext}^{1}\left(\mathcal{O}_{\mathbb{P}^{2}}, \pi_{*} \mathcal{O}_{X}\right)=0$. We may split the top row and compose the splitting with $a$ to get a diagram

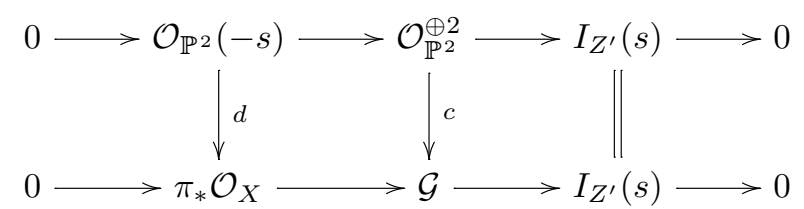

Suppose $\operatorname{Ker} c \neq 0$, then the image of $c$ is a sheaf of rank 1 , which surjects onto $I_{Z^{\prime}}(s)$. This forces that the image is isomorphic to $I_{Z^{\prime}}(s)$, which defines a splitting of the bottom row. However, since $\mathcal{G}$ is locally free, this is not possible. Thus, $\operatorname{Ker} c=0$.

Now let us consider the left vertical arrow $d: \mathcal{O}_{\mathbb{P}^{2}}(-s) \rightarrow \mathcal{O}_{\mathbb{P}^{2}} \oplus \mathcal{O}_{\mathbb{P}^{2}}(-s)$. If the cokernel is $\mathcal{O}_{\mathbb{P}^{2}}$ then we get that $\mathcal{G}$ is the trivial bundle. The only other possibility for the cokernel is $\mathcal{O}_{C} \oplus \mathcal{O}_{\mathbb{P}^{2}}(-s)$, where $C$ is a hypersurface of degree $s$ in $\mathbb{P}^{2}$. In this case, $\mathcal{G}$ sits in a sequence

$$
0 \rightarrow \mathcal{O}_{\mathbb{P}^{2}}^{\oplus 2} \rightarrow \mathcal{G} \rightarrow \mathcal{O}_{C} \oplus \mathcal{O}_{\mathbb{P}^{2}}(-s) \rightarrow 0
$$

Since $\mathcal{G}$ is a summand of $\pi_{*} \mathcal{E}$ and $H^{1}\left(\mathbb{P}^{2}, \pi_{*} \mathcal{E}\right)=0$, it follows that $H^{1}\left(\mathbb{P}^{2}, \mathcal{G}\right)=0$. This forces that

$$
H^{1}\left(\mathbb{P}^{2}, \mathcal{O}_{C}\right)=0 .
$$


But now using $0 \rightarrow \mathcal{O}_{\mathbb{P}^{2}}(-s) \rightarrow \mathcal{O}_{\mathbb{P}^{2}} \rightarrow \mathcal{O}_{C} \rightarrow 0$ we get $0=H^{1}\left(\mathbb{P}^{2}, \mathcal{O}_{C}\right)=H^{2}\left(\mathbb{P}^{2}, \mathcal{O}_{\mathbb{P}^{2}}(-s)\right)=$ $H^{0}\left(\mathbb{P}^{2}, \mathcal{O}_{\mathbb{P}^{2}}(s-3)\right)^{\vee}$, which is not possible if $s \geq 3$. Thus, the cokernel of $d$ is $\mathcal{O}_{\mathbb{P}^{2}}$ and so $\mathcal{G}$ and $\pi_{*} \mathcal{E}$ are trivial. This proves that $\mathcal{E}$ is an Ulrich bundle on $X$.

Recall from (3) that the canonical line bundle of $X$ is $\mathcal{O}_{X}(s-3)$. Since $\operatorname{det}(\mathcal{E}) \cong \mathcal{O}_{X}(s)=\mathcal{O}_{X}(s-3) \otimes$ $\mathcal{O}_{X}(3)$, it follows that $\mathcal{E}$ is a special Ulrich bundle.

Proof of Theorem 1.2. For the convenience of the reader we recall the statement from [9] that we are using. Their main result gives a description of all the possible complete intersections of codimension $r$ that can be found on a general hypersurface of degree $d$ in $\mathbb{P}^{n}$ when $2 r \leq n+2$. In our situation $n=r=2$ and $d=2 s$. In this situation the first point in [9, Theorem 5.1] enables us to conclude that for the general degree $2 s$ hypersurface $F$ we can find degree $s$ hypersurfaces $F_{1}$ and $F_{2}$ such that $F \in\left(F_{1}, F_{2}\right)$. Now we apply Theorem 2.7 and get that $X$ supports a special Ulrich bundle of rank 2. This proves Theorem 1.2.

\section{References}

[1] Marian Aprodu, Laura Costa, Rosa Maria Miró-Roig, Ulrich bundles on ruled surfaces, J. Pure Appl. Algebra 222 (1) (2018) 131-138, https://doi.org/10.1016/j.jpaa.2017.03.007.

[2] Marian Aprodu, Gavril Farkas, Angela Ortega, Minimal resolutions, Chow forms and Ulrich bundles on $K 3$ surfaces, J. Reine Angew. Math. 730 (2017) 225-249, https://doi.org/10.1515/crelle-2014-0124.

[3] Marian Aprodu, Yeongrak Kim, Ulrich line bundles on Enriques surfaces with a polarization of degree four, Ann. Univ. Ferrara, Sez. 7: Sci. Mat. 63 (1) (2017) 9-23, https://doi.org/10.1007/s11565-017-0269-z.

[4] Arnaud Beauville, Ulrich bundles on abelian surfaces, Proc. Am. Math. Soc. 144 (11) (2016) 4609-4611, https://doi.org/ $10.1090 / \mathrm{proc} / 13091$

[5] Wolf P. Barth, Klaus Hulek, Chris A.M. Peters, Antonius Van de Ven, Compact Complex Surfaces, second edition, Ergebnisse der Mathematik und ihrer Grenzgebiete. 3. Folge. A Series of Modern Surveys in Mathematics [Results in Mathematics and Related Areas. 3rd Series. A Series of Modern Surveys in Mathematics], vol. 4, Springer-Verlag, Berlin, 2004.

[6] Gianfranco Casnati, Special Ulrich bundles on non-special surfaces with $p_{g}=q=0$, Int. J. Math. 28 (8) (2017) 1750061, https://doi.org/10.1142/S0129167X17500616;

Erratum: Special Ulrich bundles on non-special surfaces with $p_{g}=q=0$, Int. J. Math. 29 (5) (2018) 1892001, https:// doi.org/10.1142/S0129167X18920015.

[7] Gianfranco Casnati, Ulrich bundles on non-special surfaces with $p_{g}=0$ and $q=1$, Rev. Mat. Complut. 32 (2) (2019) 559-574, https://doi.org/10.1007/s13163-017-0248-z;

Correction to: Ulrich bundles on non-special surfaces with $p_{g}=0$ and $q=1$, Rev. Mat. Complut. 32 (2) (2019) 575-577, https://doi.org/10.1007/s13163-019-00295-1.

[8] Gianfranco Casnati, Special Ulrich bundles on regular surfaces with non-negative Kodaira dimension, Manuscr. Math. (2021), https://doi.org/10.1007/s00229-020-01269-0.

[9] Enrico Carlini, Luca Chiantini, Anthony V. Geramita, Complete intersections on general hypersurfaces, Mich. Math. J. (57) (2008) 121-136, https://doi.org/10.1307/mmj/1220879400, special volume in honor of Melvin Hochster.

[10] Izzet Coskun, Jack Huizenga, Brill-Noether problems, Ulrich bundles and the cohomology of moduli spaces of sheaves, Mat. Contemp. 47 (2020) 21-72.

[11] David Eisenbud, Frank-Olaf Schreyer, Jerzy Weyman, Resultants and Chow forms via exterior syzygies, J. Am. Math. Soc. 16 (3) (2003) 537-579, https://doi.org/10.1090/S0894-0347-03-00423-5.

[12] Faenzi Daniele, Ulrich bundles on K3 surfaces, Algebra Number Theory 13 (6) (2019) 1443-1454, https://doi.org/10.2140/ ant.2019.13.1443.

[13] G. Horrocks, Vector bundles on the punctured spectrum of a local ring, Proc. Lond. Math. Soc. (3) 14 (1964) 689-713, https://doi.org/10.1112/plms/s3-14.4.689.

[14] J. Herzog, B. Ulrich, J. Backelin, Linear maximal Cohen-Macaulay modules over strict complete intersections, J. Pure Appl. Algebra 71 (2-3) (1991) 187-202, https://doi.org/10.1016/0022-4049(91)90147-T.

[15] Angelo Felice Lopez, On the existence of Ulrich vector bundles on some surfaces of maximal Albanese dimension, Eur. J. Math. 5 (3) (2019) 958-963, https://doi.org/10.1007/s40879-019-00336-5.

[16] Angelo Felice Lopez, On the existence of Ulrich vector bundles on some irregular surfaces, Proc. Am. Math. Soc. 149 (1) (2021) 13-26, https://doi.org/10.1090/proc/15278.

[17] P. Narayanan, A.J. Parameswaran, Ulrich line bundles on double planes, arXiv:1911.10126, 2019.

[18] Christian Okonek, Michael Schneider, Heinz Spindler, Vector Bundles on Complex Projective Spaces, Modern Birkhäuser Classics, Birkhäuser/Springer Basel AG, Basel, 2011, corrected reprint of the 1988 edition, with an appendix by S. I. Gelfand.

[19] Sheng-Li Tan, Eckart Viehweg, A note on the Cayley-Bacharach property for vector bundles, in: Complex Analysis and Algebraic Geometry, de Gruyter, Berlin, 2000, pp. 361-373. 